\title{
Cooperative Wideband Spectrum Sensing Based on Sub-Nyquist Cooperative Narrowband Spectrum Sensing in Cognitive Radio Devices
}

\author{
Evelio Astaiza ${ }^{1,2}$, Héctor Fabio Bermúdez ${ }^{2}$, Pablo Andrés Muñoz ${ }^{2}$ \\ ${ }^{1}$ GNTT - Faculty of Electronic Engineering, Universidad del Cauca (UC) \\ Popayán, Colombia \\ eastaizah@unicauca.edu.co \\ ${ }^{2}$ GITUQ - Faculty of Engineering, Universidad del Quindío (UQ) \\ Armenia, Colombia \\ \{eastaiza,hfbermudez, pamunoz\}@uniquindio.edu.co
}

\begin{abstract}
This article proposes a new cooperative wideband spectrum-sensing algorithm based on cooperative sub-Nyquist narrow band spectrum sensing in cognitive radio devices (CRD). Within this scenario, to guarantee optimal detection, the spectrum-sensing function must work with a great amount of samples of the signal obtained at rates equal or higher than the Nyquist rate, which generates high detection times, high power consumption and the need for high processing capabilities in the CRD. Additionally, we must a priori knowledge of the signal characteristics. However, in practice, the characteristics of the multiband signal are unknown and high processing capabilities would be required according to the sampling rate. Due to this, this article proposes a novel spectrumsensing algorithm for these types of systems seeking to minimize the number of samples to process and which operates without a priori knowledge of the characteristics of the multiband signal. The simulation results permit evidencing that the algorithm proposed improves the sensing performance in function of the detection probability and of the receptor's operational characteristics with respect to other cooperative wideband spectrum sensing algorithms based on sub-Nyquist sampling.
\end{abstract}

Keyword- Sub-Nyquist Sampling, Wideband Spectrum Sensing, Energy detection, Covariance Matrix.

\section{INTRODUCTION}

The electromagnetic spectrum is a limited natural resource, which, day-to-day, presents greater saturation due to the growth of the amount of devices and wireless and mobile multimedia services that require greater bandwidths. This trend has derived into the perception of shortage of the spectrum resource. However, [1] presents a report that shows that the temporal and geographic use of the spectrum assigned in the band comprised between $30 \mathrm{MHz}$ and $3 \mathrm{GHz}$ is extremely low, agreeing with the problem identified by the (US) Federal Communications Commission in 2002 [2], which establishes that the principal problem present in mobile and wireless communication systems is the underutilization of the spectrum.

Due to the aforementioned, to optimize the use of radio resources, the Cognitive Radio (CR) concept emerges [3]. It is proposed as a technology in which cognitive devices detect automatically stimuli in their radio environment and adapt intelligently their operational parameters, guaranteeing the satisfaction of the needs for communication of cognitive users (secondary users - SU) by using the spectral resources not used by the users with rights of use of the spectrum (primary users - PU). Hence, cognitive devices must be able to detect independently and efficiently the underutilized spectrum (sub-band occupied only by noise denominated white space - WS) without causing damaging interference to the PU. This capacity is denominated spectrum sensing (SS), considered one of the most critical components and enabler of CR technology.

One of the biggest problems in implementing SS is that of identifying the WS present in a multiband environment due to the large amount of samples to process by the cognitive radio device (CRD), given the high sampling rates required, increasing sensing time and the power consumed by the CRD [4]. Because of this, in recent years, based on the idea of a sparse occupation of the communications channel in multiband scenarios (i.e., few frequency bands occupied and multiple available), many proposals have been worked based on compressive sensing (CS) [5], [6]. This provides an efficient way of processing sparse signals, that is, these can be approached through expansion in terms of an adequate base, which only has some significant terms. This 
characteristic permits conducting wideband spectrum sensing by digitizing the multiband signal at rates below the Nyquist rate. Likewise, CRD require permanently performing detection of activity of the PU in the communications channel of the band of interest under conditions of signal-to-noise ratio (SNR) as low as possible (i.e., in the order of $-20 \mathrm{~dB}$ for the IEEE 802.22 standard). Within this context, one of the main challenges faced in CR is the implementation of wideband spectrum sensing (WBSS) minimizing the sampling rate required, guaranteeing high detection probability and low probabilities of miss detection and false alarm under low SNR conditions. Due to the aforementioned, seeking to improve the WBSS performance, this article proposes a novel cooperative mechanism based on the group narrowband spectrum sensing that uses subNyquist sampling. It permits minimizing the amount of samples to process, and exploit spatial diversity through the cooperation of the distinct CRD in the same region, minimizes the information load on the links with the Fusion Center (FC) (cooperative decision center) and improves performance in terms of detection and operational characteristics of the receptor compared to similar algorithms in the state-of-the-art [7][10].

In work [7] presents a cooperative wideband spectrum sensing algorithm based on two recovery algorithms of the joint support of the multiband signal from the sensing and measurement matrixes of each CRD; this implies a great load of information in the communication links between CRDs and the FC. In [8], the authors propose a cooperative wideband spectrum sensing algorithm based on the algorithm of Expectation Maximization (EM) proposed in [11] for joint estimation and detection of the spectral occupation in the multiband. The algorithm proposed operates at a sampling rate equal to or above the Nyquist rate, which implies that the CRD must process a large amount of samples, increasing sensing times and power consumed. A study [9] proposes a cooperative wideband spectrum-sensing algorithm based on sub-Nyquist multi-rate sampling and analyzes its performance in terms of the theoretical limits obtained for detection probabilities and false alarm. In [10], the authors propose a cooperative wideband spectrum sensing algorithm based on two-stage CS; the first reconstructs the multiband signal from the measurements of each CRD and the second eliminates the noise, then, decide on spectral occupation, which implies a big load of information in the communication links between the CRDs and the FC.

The rest of the article is organized as follows: section II poses the system's model; section III describes the method proposed to perform cooperative wideband spectrum sensing, based on the group narrowband spectrum sensing; section IV presents the performance evaluation of the method proposed by contrasting the evaluation metrics against those obtained through cooperative spectrum sensing methods in the state-of-the-art; and section $\mathrm{V}$ presents the study conclusions.

\section{SYSTEM MODEL}

Considering a set of $k$ groups of CRDs operating on a multiband (licenced) with a total bandwidth of $\mathrm{BHz}$, which is defined as $B=\left\{b_{1}, b_{2}, \ldots, b_{k}\right\}$ that corresponds to the set of $k$ not overlapping sub-bands of equal bandwidth $b$, equivalent to $B / k H z$ per sub-band. Each group senses a sub-band and is conformed by $q \geq k$ CDRs, as shown in Fig. 1, where each CDR is associated to a group $g_{j} \in G=\left\{g_{1}, g_{2}, \ldots, g_{k}\right\}$ with $j=1,2, . ., k$ randomly in each spatial region and according to the channel impulse response, which is assumed to follow a normal distribution with mean $\mu$, and variance $\sigma^{2}$, which is why a CDR is associated to that group presenting the best impulse response for highest correct detection probability of sub-band occupation.

Assuming that the signal samples in each sub-band is an independent random variable that follows a normal distribution of zero mean and variance $\sigma_{s}\left(N\left(0, \sigma_{s}\right)\right)$; and assuming that the noise samples in each CRD are normally distributed random variables, independent, of zero mean and variance $\sigma_{n}\left(N\left(0, \sigma_{n}\right)\right)$, the signal received by the $i$ th CRD with $i=1,2, . ., q$, in the $j$ th group (which senses the $j-t h$ sub-band) with $j=1,2, . ., k$ can be expressed as indicated in Eq. (1).

$$
\mathbf{x}_{i, j}(m)=\mathbf{h}_{i, j} \mathbf{s}_{j}(m)+\mathbf{w}_{i, j}(m)
$$

where $\mathbf{x}_{i, j}(m)$ is the $m$-th component of the signal received by the $i-t h$ CRD in the $j$-th sub-band, $h_{i, j}$ represents the cannel response for the $i-t h \mathrm{CRD}$ in the $j-t h$ sub-band, and it is assumed that $h_{i, j}$ remains constant in the sampling window ( $m$ samples), $\mathbf{s}_{j}(m)$ is the $m-t h$ component of the signal transmitted by the 
$j-t h$ PU on the $j-t h$ sub-band, and $\mathbf{w}_{i, j}(m)$ is the $m$-th noise component in the $j$-th sub-band perceived by the $i-t h$ CRD.

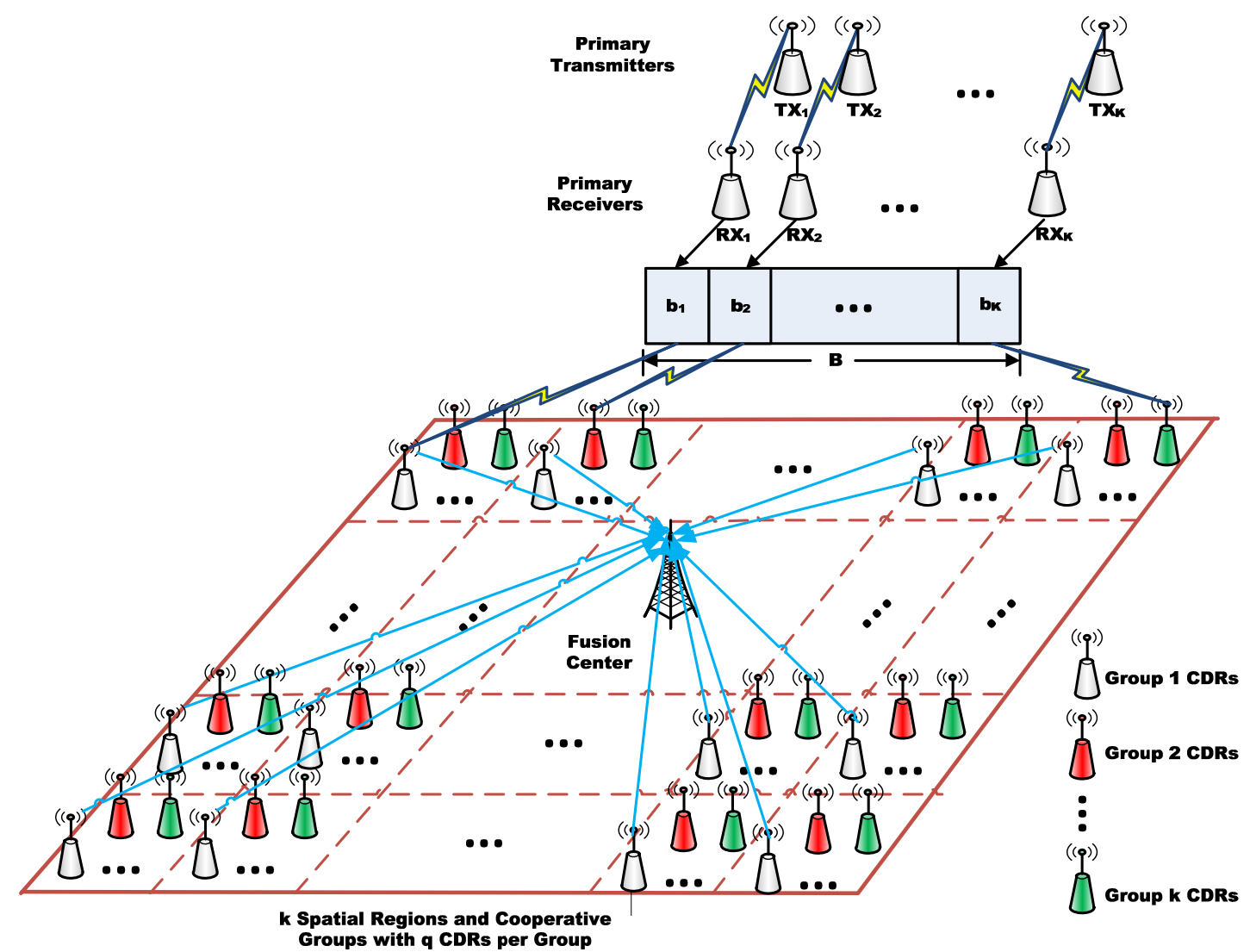

Fig. 1. Cooperative wideband spectrum sensing scenario by groups. Source: by the authors

The spectrum sensing problem in the $j-$ th sub-band may be formulated as a problem of verification of statistical hypothesis where we must choose between hypothesis $H_{0, i, j}$, which indicates that for the $i-t h \mathrm{CRD}$ the $j$-th sub-band is available, and hypothesis $H_{1, i, j}$, which indicates that for the $i-t h \mathrm{CRD}$ the $j$-th sub-band is occupied; the aforementioned can be expressed according to Eq. (2).

$$
\left\{\begin{array}{l}
H_{0, i, j}: \mathbf{x}_{i, j}=\mathbf{w}_{i, j} \\
H_{1, i, j}: \mathbf{x}_{i, j}=h_{i, j} \mathbf{s}_{j}^{*}+\mathbf{w}_{i, j}
\end{array}\right.
$$

where $\mathbf{x}_{i, j} \in \Re^{m}$ is the vector of the signal received by the $i-t h$ CRD in the $j-t h$ sub-band, with $m$ equal to the amount of samples taken per sub-band, $\mathbf{w}_{i, j} \in \mathfrak{R}^{m}$ is the vector that represents the white noise components for the $i-t h$ CRD present in the $j-t h$ sub-band, $h_{i, j} \in \Re$ is the vector that represents the channel response for the $i-t h \mathrm{CRD}$ in the $j$-th sub-band; finally, $\mathbf{s}_{j}^{*} \in \mathfrak{R}^{m}$ is the vector that represents the signal transmitted by the $j-t h \mathrm{PU}$ on the $j$-th sub-band, where the super index * denotes transposed.

\section{Proposed CoOperative Wideband Spectrum Sensing Method}

The cooperative wideband spectrum sensing method proposed is illustrated in Fig. 2. Initially, the multiband signal, $\mathbf{x}(t)$, is received by each of the CRDs. The version in each CRD corresponds to the filtered version of $\mathbf{x}(t)$ in the sub-band of interest according to the associated group, denoted by $\mathbf{x}_{i, j}(t)$ with $i=1,2, . ., q$ and 
$j=1,2, . ., k$; thereafter, the signal captured by each CRD is sampled with the Random Demodulator (RD) [12], where the sampling operation is implemented through the sampling matrix $\mathbf{A} \in \mathfrak{R}^{m \times n}$, where $m<n$ with $n$ representing the amount of signal samples when conducting the sampling at the Nyquist rate and $m$ represents the amount of Sub-Nyquist samples taken, obtaining the vector of samples $\mathbf{y}_{i, j} \in \mathfrak{R}^{m}$, where $\mathbf{y}_{i, j}=\mathbf{A} \mathbf{x}_{i, j}$. Then, the feature extraction block estimates the sparse approximation of the signal self-correlation matrix $\mathbf{R}_{x_{i, j}} \in \Re^{n \times n}$ from the covariance matrix of samples $\mathbf{R}_{y_{i, j}} \in \mathfrak{R}^{m \times m}$, where $m<n$. Then, the classification and detection block detects the occupation or not of the sub-band through the main diagonal of the estimated selfcorrelation matrix of the signal, $\mathbf{R}_{x_{i, j}} \in \mathfrak{R}^{n \times n}$; thereafter, the local decisions of each CRD are transmitted to the Fusion Center (FC), which makes the occupation decision in the sub-band by applying the OR rule among the partial decisions obtained in the previous step. Finally, the FC makes the final occupation decision in the multiband, obtaining the detection, miss detection, and false alarm probabilities in the multiband through the mean of the partial probabilities obtained in the previous step and reports the final decision of the CRD through the control channel, assumed free of errors.

The following describes the functions carried out by the blocks illustrated in Fig.2.

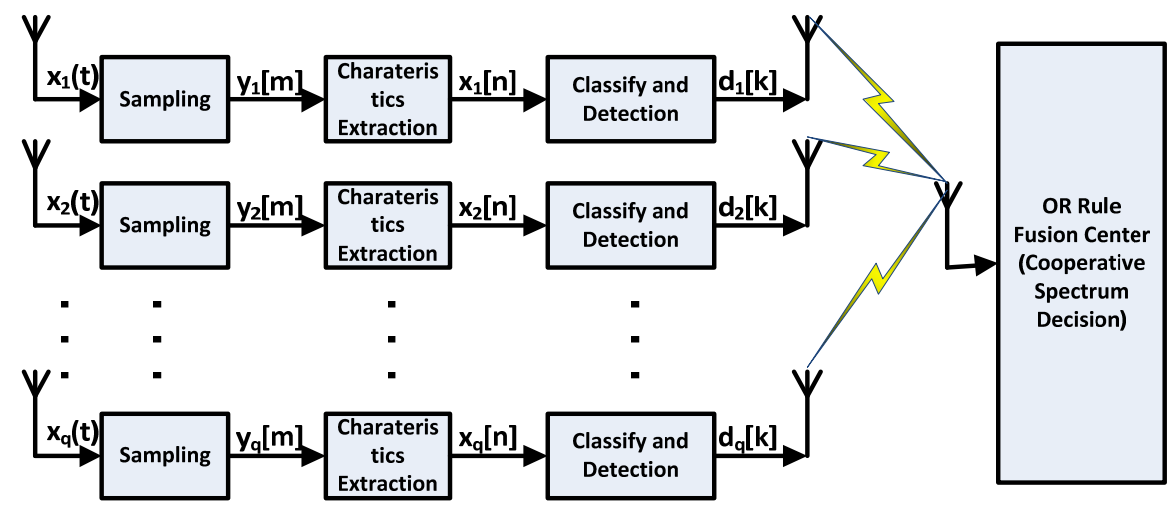

Fig. 2. Proposed cooperative wideband spectrum sensing scenario (Simplification for one sub-band - Group). Source: by the authors

\section{A. Sampling}

The multiband signal sampling, $\mathbf{x}_{i, j}(t)$, takes place through the RD and can be considered a new type of sampling system, which can be used to acquire sparse signals limited in band.

As shown in the diagram in Fig. 3, the output signal to the RD is multiplied by a high-rate pseudo-random sequence, which disperses the energy of the tones over the total bandwidth occupied by the sequence; then, antialiasing filtering is applied to finally sample the signal at a rate below the Nyquist rate. The demodulation process (multiplication by the pseudo-random sequence) guarantees that each tone present in the input signal has a different "signature" within the filter's passing band; given that the input signal to the RD is only composed of some tones, it is possible to identify the tones and their amplitudes from the low-rate samples.

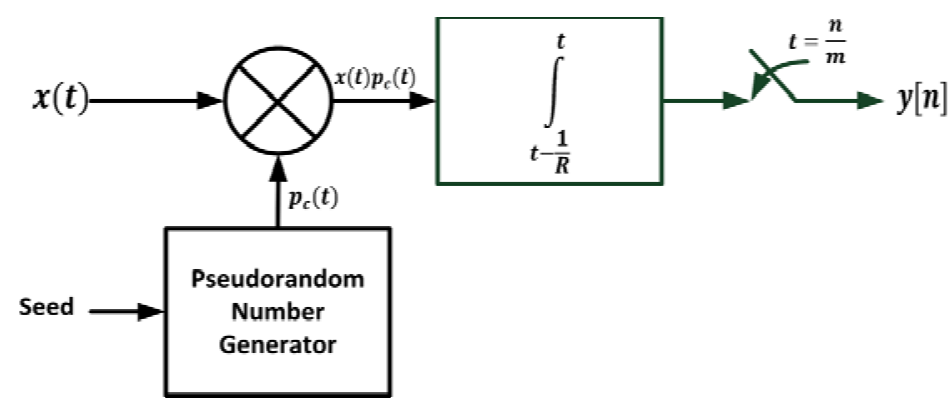

Fig. 3. Block diagram of the random demodulator.

Source: by the authors 
Samples are obtained from the sub-Nyquist sampling process, as illustrated by Eq. (3).

$$
\mathbf{y}_{i, j}=\mathbf{A} \mathbf{x}_{i, j}
$$

Where $\mathbf{A}$ is the sensing matrix of size $m \times n, \quad \mathbf{y}_{i, j} \in \mathfrak{R}^{m}$ is the measurements vector, and $\mathbf{x}_{i, j} \in \mathfrak{R}^{n}$ is the vector that represents the sparse multiband signal, $k-[6]$, hence, inputs of $\mathbf{y}_{i, j}$ are the Sub-Nyquist samples of $\mathbf{x}_{i, j}$.

\section{B. Extraction of Characteristics}

From (3), we may note that, when calculating the covariance matrix of samples, $\mathbf{R}_{y_{i, j}}$, we have the ratio given by:

$$
\mathbf{R}_{y_{i, j}}=\mathbf{A} \mathbf{R}_{\mathbf{x}_{i, j}} \mathbf{A}^{\mathbf{T}}
$$

With $\mathbf{R}_{\mathbf{x}_{i, j}}$ being the signal covariance matrix present in the communications channel of size $n \times n$ and $\mathbf{R}_{\mathbf{y}_{i, j}}$ is the covariance matrix of the samples taken with the RD of size $m \times m$.

Consequently, from the covariance matrix of the samples it is possible to obtain the signal covariance matrix in the channel and with it conduct the spectrum sensing operation, identifying the energy present in each of the $k$ sub-bands.

The spectrum-sensing function, in this order of ideas, may be conducted by identifying the values present in the principal diagonal of the estimated covariance matrix $\mathbf{R}_{\mathbf{x}_{i, j}}$, which complies Eq. (5).

$$
\mathbf{R}_{\mathbf{x}_{i, j}}(p, p)=\left\{\begin{array}{c}
2 N \sigma_{n i, j}^{2}: H_{0, i, j} \\
\left(S N R_{i, j}+N\right) \sigma_{n i, j}^{2}: H_{1, i, j}
\end{array}\right.
$$

Where $\sigma_{n i, j}^{2}$ represents the noise power in the $i-t h \mathrm{CRD}$ and $j-t h$ sub-band and $S N R_{i, j}$ denotes the signalto-noise ratio of the $i-t h \mathrm{CRD}$ in the $j-t h$ sub-band and $p=1,2, . ., m$. Then, upon identifying if Eq. (6) is fulfilled, the presence of signal can be established, which is equal to validating the hypothesis $H_{1, j}$.

$$
\mathbf{R}_{\mathbf{x}_{i, j}}(p, p)>2 N \sigma_{n i, j}^{2}
$$

To obtain the signal covariance matrix in the channel $\mathbf{R}_{\mathbf{x}_{i, j}}$ from the covariance matrix of $\mathbf{R}_{\mathbf{y}_{i, j}}$, we must solve the optimization problem Eq. (7).

$$
\min \left\|\mathbf{R}_{\mathbf{x}_{i, j}}\right\|_{1} \text { subject_to } \mathbf{R}_{y_{i, j}}=\mathbf{A} \mathbf{R}_{\mathbf{x}_{i, j}} \mathbf{A}^{\mathbf{T}}
$$

The solution proposed for (7) is an orthogonal matching pursuit (OMP) modification [13] that does not work with vectors, which is why it does not use the Kronecker product, but rather works directly in matrix form, as illustrated ahead.

Let $\quad \mathbf{x}_{i, j} \in \mathfrak{R}^{n}$ be the representation in the signal frequency domain $\mathbf{x}_{i, j}$ and $\boldsymbol{\Psi} \in \mathfrak{R}^{n \times n}$ Fourier's discrete transformation matrix, such that $\mathbf{X}_{i, j}=F\left(\mathbf{x}_{i, j}\right)=\boldsymbol{\Psi} \mathbf{x}_{i, j}$ where $\mathbf{X}_{i, j} \quad$ presents only $k \ll n$ significant values (inputs different from zero); upon sampling $\mathbf{X}_{i, j}$ with the sampling matrix $\boldsymbol{\varphi} \in \mathfrak{R}^{m \times n}$ where $k<m<n$ to obtain $\mathbf{y}_{i, j}=\boldsymbol{\varphi} \mathbf{X}_{i, j}=\boldsymbol{\varphi} \boldsymbol{\Psi} \mathbf{x}_{i, j}=\mathbf{A} \mathbf{x}_{i, j}$; if $\boldsymbol{\varphi}$ fulfills the restricted isometry property (RIP) in the order of $k$ [14], [15] and has low coherence with $\boldsymbol{\Psi}$, then $\mathbf{X}_{i, j}$ can be effectively recovered from $\mathbf{y}_{i, j}$. To conduct the estimation process of the signal covariance matrix in the channel and solve the problem posed in eq. (7), two auxiliary variables are required; the first of these $(r, s)$ to avoid the re-selection of external products. Coordinates $(r, s)$ keep the indices of the external products that can be selected; the second $\mathbf{R} \in \Re^{m \times n}$ to store the residues produced upon removing the external products selected from $\mathbf{R}_{\mathbf{y}_{i, j}}$. Initially, $\mathbf{R}$ is equal to $\mathbf{R}_{\mathbf{y}_{i, j}}$ and the $(r, s)$ variable starts with all the possible combinations of indices of external products from the columns 
of the sensing matrix $(r, s) \leftarrow\{(1,1),(1,2), \ldots,(m, n)\}$; thereafter, select the external product that best adapts to the residue through $\left(r_{t}, s_{t}\right) \leftarrow \underset{\left(r^{\prime}, s^{\prime}\right) \in(r, s)}{\arg \max } \frac{\left\langle\mathbf{R}, \mathbf{P}_{r^{\prime}, s^{\prime}}\right\rangle \mid}{\left\|\mathbf{P}_{r^{\prime}, s^{\prime}}\right\|_{2}}$, excluding from the indices those corresponding to the external product selected and calculating the weights associated to each external product selected through least squares $\hat{\mathbf{u}} \leftarrow \underset{\mathbf{u}}{\arg \min }\left\|\mathbf{R}_{\mathbf{y}_{i, j}}-\sum_{t^{\prime}=1}^{t} u_{t} \mathbf{P}_{r^{\prime}, s^{\prime}}\right\|_{2} ;$ then the residue is updated, according to the external products selected and associated weights $\mathbf{R} \leftarrow \mathbf{R}_{\mathbf{y}_{i, j}}-\sum_{t^{\prime}=1}^{t} \hat{u}_{t^{\prime}} \mathbf{P}_{r^{\prime}, s^{\prime}}$; the process is carried out in $K$ occasions to obtain the estimated covariance matrix, $\mathbf{R}_{\mathbf{x}_{i, j}}$, in which all its inputs are zero, except in the $K$ inputs corresponding to the external products selected, inputs assigned the value of the weights calculated.

In the channel covariance estimation process, we have that $\mathbf{A}=\left(\mathbf{a}_{1}, \mathbf{a}_{2}, \ldots, \mathbf{a}_{n}\right)$, where $\mathbf{a}_{r}$ is the $r-t h$ column of $\mathbf{A}$; thus, the external products are defined as $\mathbf{P}_{r, s}=\mathbf{a}_{r} \mathbf{a}_{s}{ }^{\mathbf{T}}$, permitting to represent the covariance of the samples as the weighted sum of external products, according to Eq. (8).

$$
\mathbf{R}_{\mathbf{y}_{i, j}}=\sum_{r=1}^{n} \sum_{s=1}^{n} z_{r, s} \mathbf{P}_{r, s}
$$

\section{Classification and Detection}

To detect energy for each sub-band and for each CRD, compare the energy of the signal received with a detection threshold, thus, deciding the occupation of a sub-band. To conduct this, it is necessary to obtain the values of the principal diagonal of the estimated covariance matrix of the signal, $\mathbf{R}_{\mathbf{x}_{i, j}}$, then making $\operatorname{diag}\left(\mathbf{R}_{\mathbf{x}_{i, j}}\right)=\mathbf{X}_{i, j}[f]$, the energy present in each sub-band is calculated according Eq. (9).

$$
\varepsilon_{i, j}(f)=\left|h_{i, j}\right|^{2} \sum_{j}\left|\mathbf{x}_{i, j}[f]\right|^{2}
$$

Where $\varepsilon_{i, j}$ represents the energy in the $j-t h$ sub-band received by the $i-t h \mathrm{CRD}$ over a sequence of $N$ samples, $h_{i, j}$ represents the channel response of the $i-t h$ CRD over the $j$-th sub-band, and $\mathbf{X}_{i, j}[f]$ represents the signal estimated in the $j-t h$ sub-band of the $i-t h$ CRD. Then, if the energy in the $j-t h$ sub-band of the $i-t h$ CRD is above the decision threshold $T_{h i, j}\left(\varepsilon_{i, j}>T_{h i, j}\right)$, the decision made is $H_{1, i, j}$ (occupied subband); on the contrary, the decision is $H_{0, i, j}$ (WS-free sub-band).

The detection probability $P_{d i, j}$, miss detection probability, $P_{m d_{i, j}}$, and false alarm probability, $P_{f_{i, j}}$, for the $i-t h \mathrm{CRD}$ in the $j$-th sub-band are defined as indicated in Eqs. (10), (11), and (12).

$$
\begin{gathered}
P_{d i, j}=P\left(H_{1, i, j} \mid H_{1, i, j}\right) \\
P_{m d_{i, j}}=P\left(H_{0, i, j} \mid H_{1, i, j}\right)=1-P_{d_{i, j}} \\
P_{f_{i, j}}=P\left(H_{1, i, j} \mid H_{0, i, j}\right)
\end{gathered}
$$

Understanding as detection probability that correct detection probability of occupation of a sub-band or of presence of signal of a PU in a sub-band (decide $H_{1, i, j}$ when $H_{1, i, j}$ is true); the probability of false alarm is understood as the probability associated to detecting signal of a PU when in a sub-band there is actually white space (decide $H_{1, i, j}$ when $H_{0, i, j}$ is true). The probability of miss detection is understood as that probability associated to detecting a white space when in a sub-band there is the presence of signal of a PU (decide $H_{0, i, j}$ when $H_{1, i, j}$ is true).

According to the central limit theorem [16], if the number of samples is sufficiently large ( $\geq 10$ in practice), the statistics (mean and variance) of $\varepsilon_{i, j}$ associated to hypotheses $H_{0, i, j}$ and $H_{1, i, j}$ are normally distributed asymptotically and given by Eqs. (13) and (14). 


$$
\begin{aligned}
E\left(\varepsilon_{i, j}\right) & =\left\{\begin{array}{l}
2 N \sigma_{n i, j}^{2}: H_{0, i, j} \\
\left(S N R_{i, j}+N\right) \sigma_{n i, j}^{2}: H_{1, i, j}
\end{array}\right. \\
\operatorname{Var}\left(\varepsilon_{i, j}\right) & =\left\{\begin{array}{l}
2 N \sigma_{n i, j}^{4}: H_{0, i, j} \\
2\left(2 S N R_{i, j}+N\right) \sigma_{n i, j}^{4}: H_{1, i, j}
\end{array}\right.
\end{aligned}
$$

With $\sigma_{n i, j}^{2}$, which denotes the noise energy in the $i-t h \mathrm{CRD}$ and sub-band and $S N R_{i, j}$ denotes the signal-tonoise ratio of the $i-t h$ CRD in the $j-t h$ sub-band.

Then, the detection and false alarm probabilities of the $i-t h$ CRD in the $j-t h$ sub-band can be expressed as indicated in Eqs. (15) and (16).

$$
\begin{aligned}
& P_{d i, j}=Q\left[\frac{T_{h i, j}-E\left({ }_{i, j} \mid H_{1, j}\right)}{\sqrt[2]{\operatorname{Var}\left({ }_{i, j} \mid H_{1, j}\right)}}\right]=Q\left[\frac{T_{h i, j}-\left(S N R_{i, j}+N\right) \sigma_{n i, j}^{2}}{\sqrt[2]{2\left(2 S N R_{i, j}+N\right) \sigma_{n i, j}^{4}}}\right] \\
& P_{f_{i, j}}=Q\left[\frac{T_{h_{i, j}}-E\left({ }_{i, j} \mid H_{0, j}\right)}{\sqrt[2]{\operatorname{Var}\left({ }_{i, j} \mid H_{0, j}\right)}}\right]=Q\left[\frac{T_{h_{i, j}}-2 N \sigma_{n i, j}^{2}}{\sqrt[2]{2 N \sigma_{n i, j}^{4}}}\right]
\end{aligned}
$$

Where

$$
Q(x)=\frac{1}{\sqrt{2 \pi}} \int_{x}^{\infty} e^{\frac{-t^{2}}{2}} d t
$$

Hence, the decision threshold, $T_{h i, j}$, for a specific value of $P_{f_{i, j}}$ is given by (18).

$$
T_{h i, j}=Q^{-1}\left(P_{f_{i, j}}\right) \sqrt[2]{2 N \sigma_{n i, j}^{4}}+2 N \sigma_{n i, j}^{2}
$$

\section{Cooperative Decision}

Afterward, proceed to jointly decide the occupation per sub-band through OR rule among the preliminary decisions of each CRD. According to the OR decision rule, when at least in one of the $q$ versions of the subband (one version per CRD) occupation is detected, the final decision is that the sub-band is occupied. Hence, the detection and false alarm probabilities per final sub-band are expressed according to Eqs. (19) and (20).

$$
\begin{aligned}
& P_{d_{j}}=1-\prod_{i=1}^{q}\left(1-P_{d_{i, j}}\right) \\
& P_{f_{j}}=1-\prod_{i=1}^{q}\left(1-P_{f_{i, j}}\right)
\end{aligned}
$$

Thereby, the detection probability, $P_{d j}$, miss detection probability, $P_{m d_{j}}$, and false alarm probability, $P_{f j}$, in the $j$-th sub-band are defined according to Eqs. (21), (22), and (23).

$$
\begin{gathered}
P_{d_{j}}=1-\prod_{i=1}^{q}\left(1-Q\left[\frac{T_{h i, j}-\left(S N R_{i, j}+N\right) \sigma_{n i, j}^{2}}{\sqrt[2]{2\left(2 S N R_{i, j}+N\right) \sigma_{n i, j}^{4}}}\right]\right) \\
P_{m d_{j}}=\prod_{i=1}^{q}\left(1-Q\left[\frac{T_{h_{i, j}-\left(S N R_{i, j}+N\right) \sigma_{n i, j}^{2}}^{2}}{\sqrt[2]{2\left(2 S N R_{i, j}+N\right) \sigma_{n i, j}^{4}}}\right]\right) \\
P_{f_{j}}=1-\prod_{i=1}^{q}\left(1-Q\left[\frac{T_{h_{i, j}}-2 N \sigma_{n i, j}^{2}}{\sqrt[2]{2 N \sigma_{n i, j}^{4}}}\right]\right)
\end{gathered}
$$

Finally, calculate the detection probabilities, $P_{d}$, miss detection probability, $P_{m d}$, and false alarm probability, $P_{f}$, of the multiband according to Eqs. (24), (25), and (26). 


$$
\begin{gathered}
P_{d}=\frac{1}{K} \sum_{j=1}^{K}\left\{1-\prod_{i=1}^{q}\left(1-Q\left[\frac{T_{h_{i, j}}-\left(S N R_{i, j}+N\right) \sigma_{n i, j}^{2}}{\sqrt[2]{2\left(2 S N R_{i, j}+N\right) \sigma_{n i, j}^{4}}}\right]\right)\right\} \\
P_{m d}=\frac{1}{K} \sum_{j=1}^{K}\left\{\prod _ { i = 1 } ^ { q } \left(1-Q\left[\frac{T_{h_{i, j}-\left(S N R_{i, j}+N\right) \sigma_{n i, j}^{2}}^{2}}{\left.\left.\left.\sqrt[2]{2\left(2 S N R_{i, j}+N\right) \sigma_{n i, j}^{4}}\right]\right)\right\}}\right.\right.\right. \\
P_{f}=\frac{1}{K} \sum_{j=1}^{K}\left\{1-\prod_{i=1}^{q}\left(1-Q\left[\frac{T_{h_{i, j}}-2 N \sigma_{n i, j}^{2}}{\sqrt[2]{2 N \sigma_{n i, j}^{4}}}\right]\right)\right\}
\end{gathered}
$$

\section{E. Proposed Algorithm}

To implement spectrum sensing, according to the process described in literals A, B, C, and D, the algorithm illustrated in Table I is proposed to decide on the spectrum occupation in each CDR. The algorithm's input parameters are: the sensing matrix, $\mathbf{A}$, the channel's samples vector, $\mathbf{y}_{i, j}$, the size of the samples vector, $m$, and the size of the signal vector, $n$, (line 1). The algorithm proposed in each CRD returns the decision of occupation or not of the sensed sub-band, ch, (line 2). Use the auxiliary variable, Pc, to store the power in the sub-band sensed (line 3). The spectrum sensing process starts by calculating the average number of components required to conduct the sensing (line 5), then, the covariance matrix is estimated through the covariance estimation function described in literal B of this section (line 6). Afterward the vector of the principal diagonal is obtained from the estimated covariance matrix (line 8) that represents the power of the components estimated from the signal, then, calculate the power estimated in the sub-band (line 9); finally, estimate and return the presence or not of signal in the sub-band sensed, ch, (lines 11 to 16).

TABLE I

Narrowband Spectrum Sensing Algorithm, NBCS_CDR, for each CDR.

\begin{tabular}{|ll|}
\hline \multicolumn{1}{|c|}{ Algorithm: NBCS_CDR } \\
\hline 1 & Input: $\mathbf{A}, \mathbf{y}_{i, j}, m, n$ \\
2 & Output: $\mathbf{c h}$ \\
3 & Var: $\quad \mathbf{P c}=0 \%$ Power Signal Components \\
4 & Start \\
5 & $K=(n / m)$ \\
6 & $\mathbf{R}_{\mathbf{x}} \leftarrow$ Covariance_Estimation $\left(\mathbf{A}, \mathbf{y}_{i, j}, K\right)$ \\
7 & For $i=1$ until $n$ \\
8 & $\quad \mathbf{X}(i) \leftarrow \mathbf{R}_{x}(i, i)$ \\
9 & $\mathbf{P c} \leftarrow \mathbf{P c}+|\mathbf{X}(i)|^{2}$ \\
10 & End Stop \\
11 & If $\mathbf{P c}>0$ then \\
12 & $\mathbf{c h} \leftarrow 1$ \\
13 & If not \\
14 & $\mathbf{c h} \leftarrow 0$ \\
15 & End if \\
16 & Return $\mathbf{c h}$
\end{tabular}


TABLE II

Cooperative Group Wideband Sensing Spectrum (CGWBSS) in FC.

\begin{tabular}{|ll|}
\hline & \multicolumn{1}{c|}{ Algorithm: CGWBSS } \\
\hline 1 & Input: $\mathbf{C H}$ \\
2 & Output: $\boldsymbol{O} c_{-} \mathbf{M b}$ \\
3 & Start \\
4 & For $i=1$ until $k$ \\
5 & $\quad$ Oc_Mb(i) $=\mathbf{C H}(i, 1)$ OR $\mathbf{C H}(i, 2) \ldots O R \mathbf{C H}(i, q)$ \\
6 & End Stop \\
7 & Return $O \boldsymbol{c}_{-} \mathbf{M b}$ \\
\hline
\end{tabular}

Once all the CRDs in the cognitive network sense the associated sub-band, these report the individual decisions to the FC, where the algorithm illustrated in Table II is implemented. The input parameter is the decision matrix of the $\mathrm{CDR}, \boldsymbol{C H} \in \mathfrak{R}^{k \times q}$, where each row corresponds to the decisions sent by the CRD belonging to a group (line 1); the algorithm proposed returns to the vector of sub-bands occupied and available in the multiband $\boldsymbol{O} \boldsymbol{c} \_\boldsymbol{M} \boldsymbol{b}$ (line 2); then, makes the decision to occupy each sub-band through OR rule among the decisions made by the CRDs belonging to each group (line 5). Finally, we obtain and return the multiband occupation decision vector $\boldsymbol{O} \boldsymbol{c} \_\boldsymbol{M b}$ (lines 4 to 7).

\section{IV.PERFORMANCE EVALUATION}

\section{A. Scenario and Simulation Parameters}

In implementing the simulation of the cooperative wideband spectrum sensing algorithm proposed, a multiband signal is generated, according to the simulation parameters shown in Table III.

TABLE IIII

Simulation parameters

\begin{tabular}{|c|c|}
\hline Parameter & Value \\
\hline \multicolumn{2}{|c|}{ Multiband Signal Generation } \\
\hline Multiband Signal Bandwidth, $B W$ & $20 \mathrm{MHz}$ \\
\hline Bandwidth per Channel, $B$ & $3.3 \mathrm{MHz}$ \\
\hline Signal Modulation in Channel & 16 QAM \\
\hline Symbol Rate, $R s$ & $2 \mathrm{Msps}$ \\
\hline Roll Off Transmission Filter Factor, $\rho$ & 0.5 \\
\hline Nyquist Sampling Rate, $f s$ & $40 \mathrm{MHz}$ \\
\hline $\begin{array}{l}\text { Number of Multiband Signal Samples } \\
\text { (Nyquist), } n\end{array}$ & 2000 \\
\hline Carrier Frequencies, $f c$ & $\begin{array}{l}3 \mathrm{MHz}, \\
6 \mathrm{MHz}, \\
9 \mathrm{MHz}, \\
12 \mathrm{MHz}, \\
15 \mathrm{MHz} \text { and } \\
18 \mathrm{MHz}\end{array}$ \\
\hline \multicolumn{2}{|c|}{ Sub-Nyquist Sampler } \\
\hline Number of Input Signal Samples, $n$ & 2000 \\
\hline Number of Input Signal Samples, $m$ & 100 \\
\hline \multicolumn{2}{|c|}{ Sensing Algorithm CWBSS } \\
\hline Number of Cooperative Users, $q$ & 2 \\
\hline
\end{tabular}

\section{B. Simulation results, Metrics, Characterization, and Comparison}

To assess the performance of the spectrum-sensing algorithm proposed, use the detection probability as metrics, analyzed in function of the multiband signal-to-noise ratio generated and the receptor operational characteristics (ROC) curve, compared to the metrics obtained from the algorithms proposed in [7]-[10]; the results obtained are shown in Figs. 4 to 7. 
Fig. 4 shows the performance of the algorithm proposed against the performance of the algorithms of cooperative wideband spectrum sensing proposed in [7]-[10]. The figure illustrates that the performance of the algorithms in [7]-[10] is lower than that reached by the algorithm proposed, in function of the detection probability. It is, likewise, noted that the detection probability for the algorithm proposed is approximately equal to 1 for SNR values above $-5 \mathrm{~dB}$.

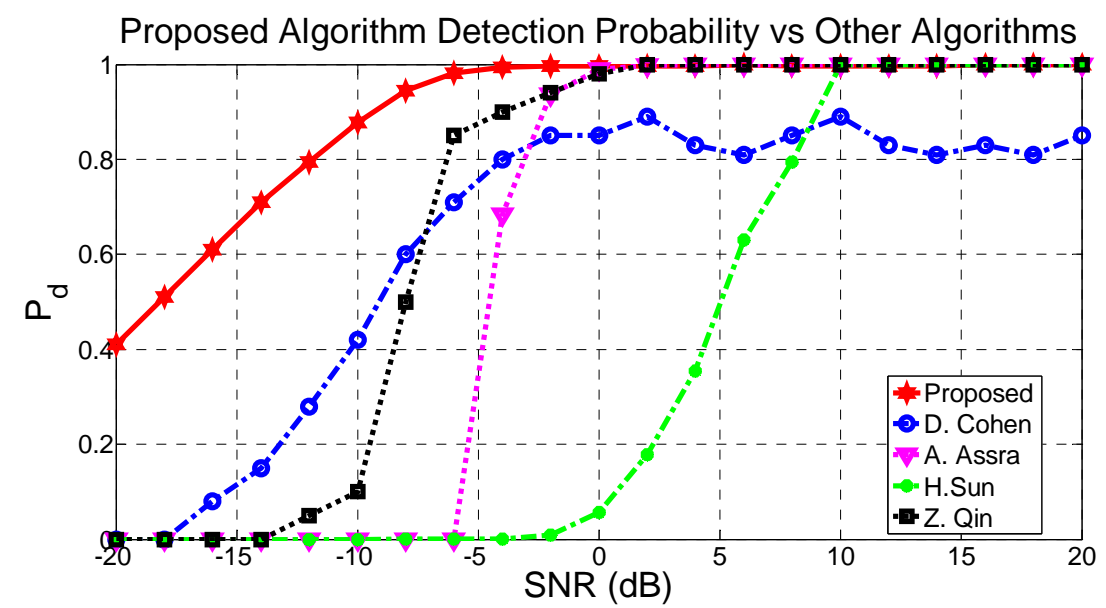

Fig. 4. Performance of cooperative algorithm proposed vs. other cooperative sensing algorithms in function of SNR. Source: by the authors

Fig. 5 shows that the best performance in terms of ROC curves corresponds to the algorithm proposed; this is because the area under the curve of the algorithm proposed is the biggest, which indicates the capacity of the algorithm proposed to identify WS positively. As also noted in Fig. 5, the algorithm with the worse performance is that proposed by Sun [9], given that the ROC curve covers a smaller area than the corresponding to the other algorithms. Considering that the results illustrated in Fig. 5 correspond to the ROC curves of the algorithms contrasted from a SNR of $-3 \mathrm{~dB}$, again we note that the algorithm proposed improves significantly the performance of the other algorithms under conditions of low SNR.

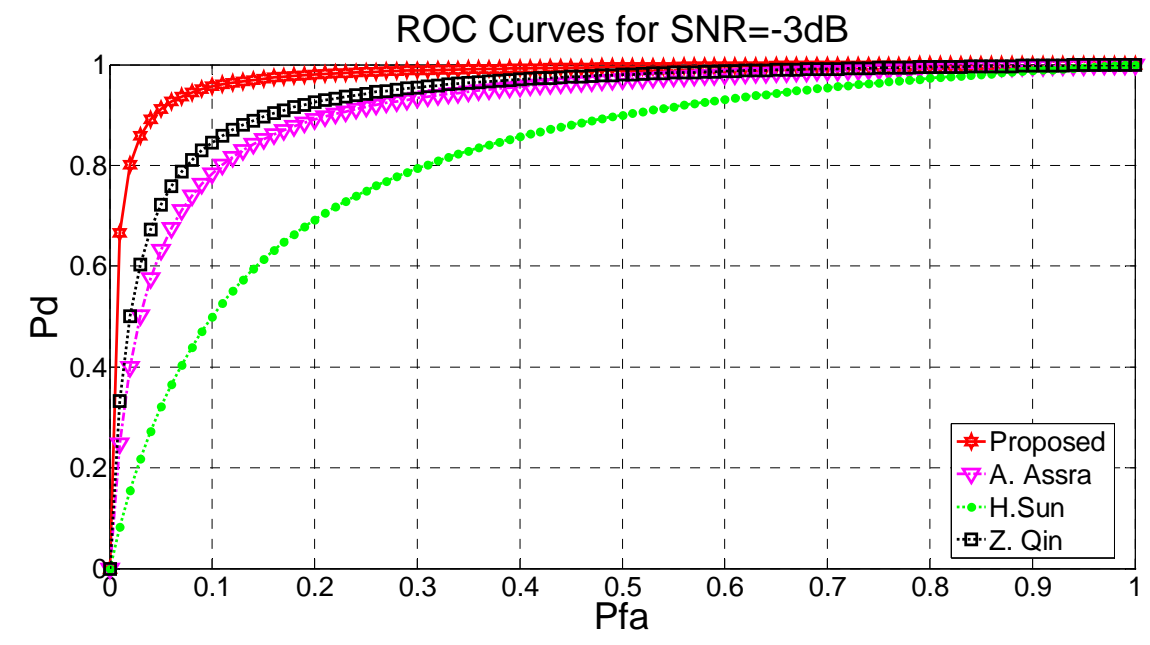

Fig. 5. ROC curves for $\mathrm{SNR}=-3 \mathrm{~dB}$

Source: by the authors

Fig. 6 illustrates the performance of the algorithm proposed in function of the detection probability against SNR, according to the number of CRDs cooperating in a group. Herein is noted that as the amount of CRDs cooperating is greater, the SNR at which a detection probability is reached approximately equal to one diminishes, achieving the target performance in CR for the 802.22 standard with an approximate amount of 20 CRDs cooperating in each group. 


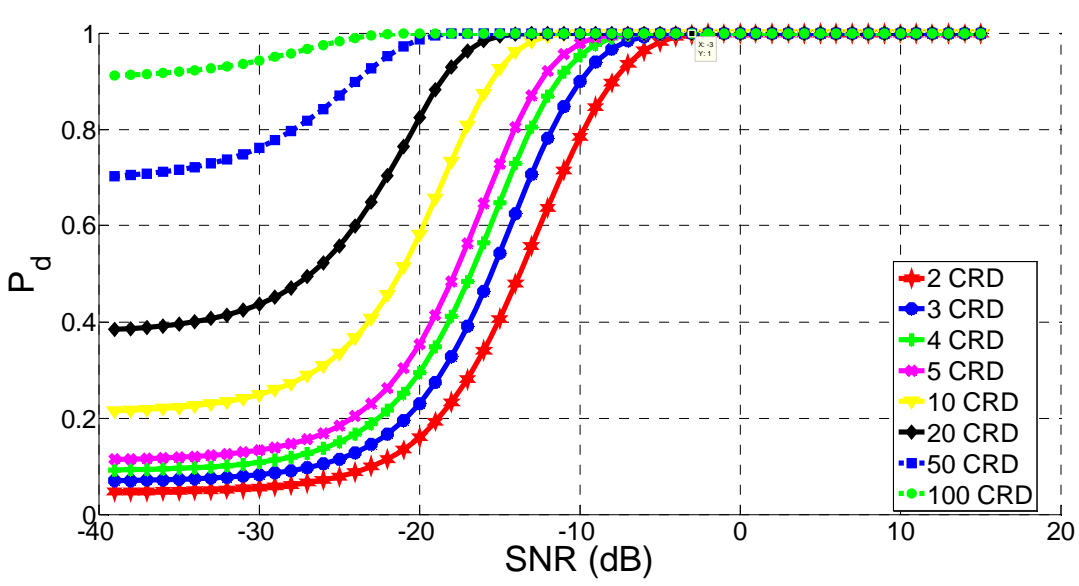

Fig. 6. Detection probability against SNR in function of the number of CRDs cooperating per group (Sub-bands $=6$ ). Source: by the authors

Fig. 7 shows the performance of the algorithm proposed in function of the detection probability against SNR, according to the number of sub-bands defined in the multiband sensed. Therein, it is possible to evidence that as a higher number of sub-bands exist in the multiband, performance of the algorithm diminishes.

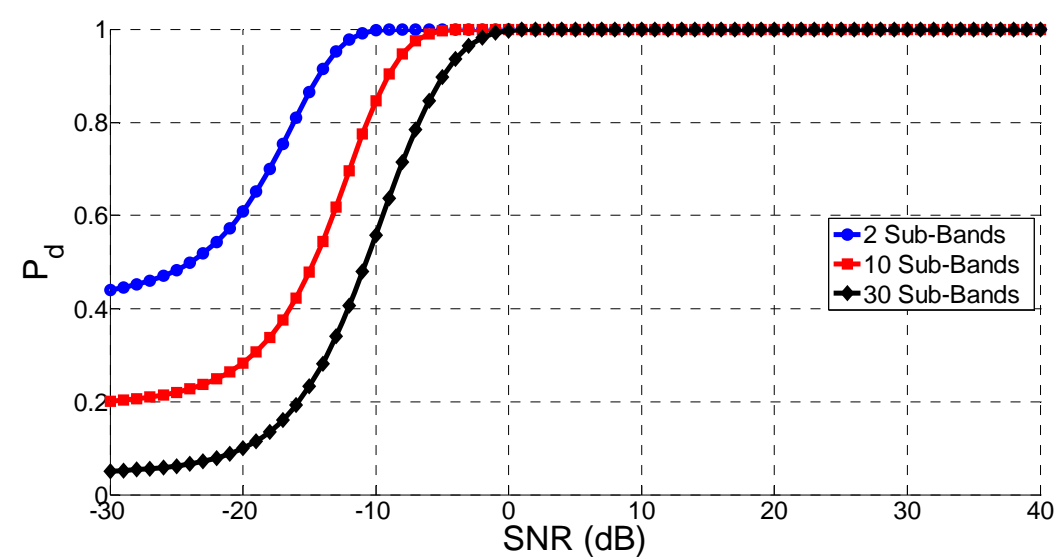

Fig. 7. Detection probability against SNR in function of the number of sub-bands in the multiband (Cooperating CRDs $=3$ per group). Source: by the authors

\section{Computational Complexity Analysis}

The computational complexity of the algorithm proposed can be analyzed in three stages. The first stage corresponds to the sub-sampling carried out with $\mathrm{RD}$, which requires an amount of $\mathrm{Km}$ scalar multiplications [4]. The second stage, which calculates the covariance matrix of the samples, obtained from $m^{2}$ scalar multiplications; thereafter, the signal covariance matrix is reconstructed. The algorithm described in section $3 \mathrm{~B}$ identifies that the projection operation is limited by $O\left(m n^{2}\right)$. Given that $m<n$, the selection operation of the best external product has complexity $O\left(n^{2}\right)$ because $n^{2}$ external products exist. The weight selection operation has complexity $O\left(\mathrm{~m}^{2}\right)$, given that the highest amount of operations is represented by the product of matrices sized $(m \times m)$ of the internal product associated to the problem of least squares optimization and finalizing this stage we find the residue update operation, which presents complexity $O\left(K^{2}\right)$. In conclusion, given that the maximum complexity of the operations conducted in this stage is that associated to the projection operation, then the complexity of the complete stage is $O\left(m n^{2}\right)$. The third and last stage corresponds to the sensing consolidation in which, as illustrated in Tables I and II, the signal covariance estimation operation (stage 2) presents complexity $O\left(m n^{2}\right)$, then lines 7 to 15 from Table I have complexity $n+1$ and, given that $n>k$ the complexity may be defined as $O(n)$. Finally, the lines from Table II have complexity $k$ with which it is defined as complexity of the third stage $O\left(m n^{2}\right)$. 
In synthesis, the computational complexity of the three stages that comprise the method proposed will be the maximum complexity of the three prior stages $O\left(m n^{2}\right)=\max \left[\operatorname{Km}, O\left(m n^{2}\right), O\left(m n^{2}\right)\right]$.

\section{CONCLUSIONS}

This article proposed a novel cooperative wideband spectrum-sensing algorithm in CR devices based on the conformation of narrowband sensing groups, demonstrating that through the algorithm proposed it is possible to perform the wideband spectrum sensing function by using an amount of samples smaller than those obtained at the Nyquist rate. This allowed reaching performance above other cooperative spectrum sensing algorithms proposed in the state-of-the-art. Additionally, it permits performing the wideband spectrum sensing function efficiently and by complying with the CR requirements with respect to reliable detection in low SNR conditions.

\section{REFERENCES}

[1] M. McHenry, "NSF spectrum occupancy measurements project summary," Shared Spectrum Company, Tech. Rep., Aug. 2005.

[2] Spectrum Policy Task Force Report, FCC ET Docket 02-155, 2002.

[3] J. Mitola, "Cognitive radio: An integrated agent architecture for software defined radio," Doctor of Technology, Royal Inst. Technol. (KTH), Stockholm, Sweden, 2000.

[4] M. Mishali, Y. C. Eldar, O. Dounaevsky and E. Shoshan, "Sampling: Analog to Digital at Sub-Nyquist Rates", IET Journals \& Magazines, vol. 5, No. 1, pp. 8-20, 2011.

[5] R. Baraniuk, "Compressive sensing”, IEEE Signal Processing Magazine, pp. 118-121, July 2007.

[6] E. Astaiza Hoyos, P.E. Jojoa Gómez, H.F. Bermúdez Orozco, "Compressive sensing: a methodological approach to an efficient signal processing", Revista DYNA, pp. 203 - 210, Aug. 2015.

[7] D. Cohen, A. Akiva, B. Avraham and Y.C. Eldar, "Centralized Cooperative Spectrum Sensing from Sub-Nyquist Samples for Cognitive Radios" in IEEE Cognitive Radio and Networks Symposium, pp. 7486-7491, 2015.

[8] A. Assra, J. Yang and B. Champagne, "An EM Approach for Cooperative Spectrum Sensing in Multiantenna CR Networks," in IEEE Transactions On Vehicular Technology, pp. 1229 - 1243, 2016.

[9] H. Sun, A. Nallanathan, S. Cui and C.X. Wang, "Cooperative Wideband Spectrum Sensing Over Fading Channels," IEEE Transactions on Vehicular Technology, pp. 1382-1394, 2016.

[10] Z. Qin, Y. Gao, M.D. Plumbley and C.G. Parini, "Wideband Spectrum Sensing on Real-Time Signals at Sub-Nyquist Sampling Rates in Single and Cooperative Multiple Nodes", in IEEE Transactions On Signal Processing, pp. 3106 - 3117, 2016.

[11] Q. Guo and D. Huang, "EM-based joint channel estimation and detection for frequency selective channels using Gaussian message passing," IEEE Trans. Signal Process., vol. 59, no. 8, pp. 4030-4035, Aug. 2011.

[12] J. A. Tropp, J. N. Laska, M. F. Duarte, J. K. Romberg, and R. G. Baraniuk, "Beyond Nyquist: Efficient sampling of sparse band limited signals," IEEE Trans. Information Theory, vol. 56, no.1, pp. 520-544, Jan. 2010.

[13] J. Tropp and A. Gilbert, "Signal recovery from random measurements via orthogonal matching pursuit," IEEE Trans. Inform. Theory, vol. 53, no. 12, pp. 4655-4666, DEq. 2007.

[14] M. Mishali and Y. C. Eldar, "Blind multiband signal reconstruction: Compressed sensing for analog signals," IEEE Trans. Signal Process., vol. 57, no. 3, pp. 993-1009, Mar. 2009.

[15] C.-P. Yen, Y. Tsai, and X. Wang, "Wideband spectrum sensing based on sub-Nyquist sampling," IEEE Trans. Signal Process. vol. 61, no. 12, pp. 3028-3040, Jun. 2013.

[16] B. V. Gendenko and A. N. Kolmogorov, "Limit Distributions for Sums of Independent Random Variables", Reading, MA: AddisonWesley, 1954.

\section{AUTHOR PROFILE}

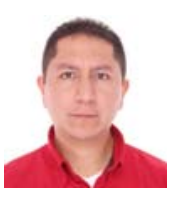

E. Astaiza-Hoyos, Electronics Engineer from Universidad of Cauca (1998). Masters in Engineering, area of Telecommunications, Universidad of Cauca (2008). Member IEEE. Currently PhD candidate sciences of Electronics. Associate Professor at Universidad of Quindío, program of Electronic Engineering, Researcher with the Telecommunications Research group (GITUQ) at Universidad of Quindío. Areas of interest: wireless communications, spectrum sensing.

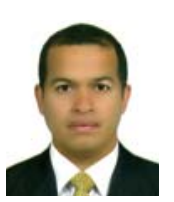

H. F. Bermúdez-Orozco, Electronics Engineer from Universidad of Cauca (2000). Masters in Electronics and Telecommunications, Universidad of Cauca (2010). Member IEEE. Current PhD student in Telematic Engineering. Associate Professor at Universidad of Quindío, program of Electronic Engineering, Coordinator of the Telecommunications Research group (GITUQ) at Universidad of Quindío. Areas of interest: wireless communications, radiant systems and propagation, modeling of traffic of telematics services.

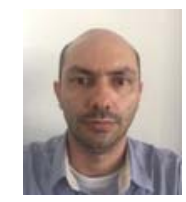

P. A. Muñoz-Gutierrez, Electronics Engineer from National University of Colombia Campus Manizales (2000). Masters in Electric Engineering, Technological University of Pereira (2006). Assistant Professor at University of Quindío, program of Electronic Instrumentation Technology, Coordinator of the Automatic and Machine Learning Group (GAMA) at University of Quindío. Areas of interest: Automatic Control, Inverse Problems and Regularization, Machine Learning. 